\title{
Velocity-dependent improvements in single-dot direction discrimination
}

\author{
NESTOR MATTHEWS and LESLIE WELCH \\ Brown University, Providence, Rhode Island
}

\begin{abstract}
Thirty-six Brown University students participated in three experiments designed to address perceptual learning. In each experiment, visual discrimination thresholds were tracked over 4,200 trials. Results from Experiment 1 suggest that the pattern of threshold reduction on a single-dot motion-direction discrimination task was stimulus-direction specific and matched (in a velocity-dependent manner) the threshold reduction pattern previously reported for a line-orientation discrimination task. In Experiment 2, it was determined that the stationary-line-orientation-specific practice effects originally reported by Vogels and Orban (1985) could be replicated but were contingent on line length. Similarly, the results from Experiment 3 suggest that practice effects originally reported by Ball and Sekuler (1987) could be replicated but were contingent on stimulus velocity. Implications for the mechanisms underlying direction and orientation discrimination are considered.
\end{abstract}

The purpose of this study was to investigate how certain kinds of visual experience affect visual performance. To that end, perceptual learning - a decrease in visual threshold caused by the repetition of a visual task - was assessed. Many researchers in this area also refer to perceptual learning as practice effects, or practice-based improvements. Practice effects are typically considered an undesirable confounding variable in many experimental settings, and many studies are conducted only after the psychophysical observer has "overcome" the practice effects and demonstrates stable performance. While these concerns are legitimate within the context of many experimental questions, the characteristics of the practice effects can provide valuable clues about the visual mechanisms that mediate various visual functions.

Several perceptual learning studies have addressed a well-known visual phenomenon, the oblique effect (Ball \& Sekuler, 1987; Vogels \& Orban, 1985). The oblique effect (Appelle, 1972) is a reduced sensitivity to visual stimuli that are neither vertical nor horizontal (cardinal) in orientation or direction of motion. The question addressed in these studies was whether the oblique effect could be overcome with ample visual experience. In one study, Vogels and Orban (1985) examined the effect of practice on the oblique effect in line-orientation tasks. In another investigation, Ball and Sekuler (1987) sought to understand the differences in perceptual learning that characterize oblique and cardinal random-dot direction discrimination. As a review of each will show, there are important differences in practice effects for these two different visual tasks.

A subset of this work was presented at the 1995 ARVO meeting. The authors would like to thank Robert Sekuler for helpful discussions, and Mary Hayhoe and two anonymous reviewers for their suggestions on the manuscript. Correspondence should be addressed to N. Matthews, Department of Psychology, Brown University, 89 Waterman St., Providence, RI 02912 (e-mail: nestor_matthews@brown.edu).
The Vogels and Orban (1985) study addressed the issue of whether orientation discrimination thresholds could be lowered with practice and, if so, whether such practice effects are orientation specific. Each observer was assigned to one orientation and trained at that orientation for at least 5,000 trials (in biocks of 60 trials). Just noticeable differences (JNDs) in orientation were measured for each observer during the course of the experiment. The results showed that despite a nearly $50 \%$ decline in NDs for all observers in the oblique conditions, the JNDs for oblique stimuli were greater at the completion of training than the JNDs for cardinally oriented stimuli before training. It was also found that training at cardinal orientations resulted in no reduction in JNDs. Thus, the results of their experiment suggest that improvements in orientation discrimination are possible, but only for oblique stimuli, and that the oblique effect in orientation persists even after 5,000 practice trials. But, would the pattern of learning that characterizes line-orientation discrimination also be found in direction discrimination tasks?

In a series of experiments, Ball and Sekuler (1987) investigated direction-specific improvements in random-dot motion discrimination. At issue in that study was whether the direction of motion differentially affected discrimination performance. Their results suggested that direction discrimination is better for faster velocities than for slower velocities and can be improved with practice at both oblique and cardinal directions of motion. Comparable levels of perceptual learning were found at the oblique and cardinal directions. Ball and Sekuler (1987) also reported that, with selective training, final oblique thresholds can become lower than initial cardinal thresholds.

The findings from the stationary-line-orientation studies and moving-random-dot direction studies differ in several ways. First, while practice significantly improved performance on both direction and orientation tasks for oblique stimuli, significant improvements in cardinal dis- 
crimination occurred only in the random-dot direction task. Second, the practice effects associated with the oblique and cardinal conditions in the random-dot direction task did not differ from each other. By contrast, training on the oblique orientation discrimination task produced both a significant decline in JND (i.e., a nonzero practice effect) and significantly greater threshold reduction than that observed on the cardinal orientation discrimination task. Thus, differential perceptual learning was evident on the orientation discrimination task, but not on the random-dot direction discrimination task. Third, over the course of training, there was some overlap between performance levels on the oblique and cardinal random-dot motion discrimination tasks. This was not the case on the orientation discrimination task, where even the smallest oblique JNDs were larger than any JNDs obtained in the cardinal condition.

That the results from these two studies are dissimilar is unsurprising, given the physical differences between the stimuli on which the observers practiced. Vogels and Orban (1985) presented single stationary lines subtending $15^{\circ}$ of visual angle for $600 \mathrm{msec}$, whereas Ball and Sekuler's (1987) moving random dots traversed a $5^{\circ}$ trajectory over a duration of $500 \mathrm{msec}$. Even if the presentation duration had been constant between the two studies, the dissimilar patterns of perceptual learning might still be attributable to the difference between the random dots' trajectory length and the length of the stationary lines. There is some evidence that the orientation-related oblique effect in cats diminishes as line length decreases (Vandenbussche, Orban, \& Maes, 1983; Vogels, Orban, \& Vandenbussche, 1984). The dissimilar practice effects observed in these two studies may also have been due to the fact that a given orientation was represented at just one spatial location for each presentation of the stationary line stimulus but at several hundred spatial locations for each presentation of the random-dot display. That is, each of the dots in a coherently moving random-dot pattern effectively "paints" a trajectory that has an orientation identical to the global direction. Thus, one cannot eliminate a priori the possibility that the dissimilarities in the practice effects between the two studies were caused by differences in the number of cues to the stimulus' direction/orientation. However, the most apparent explanation of the dissimilar practice effects is that a random-dot pattern moves, whereas a stationary line does not. The practice effects may simply be dependent on the presence or absence of motion.

In the present study, we attempted to distinguish among these various possibilities to gain a greater understanding of the mechanism(s) underlying orientation and direction discrimination. It was therefore necessary to assess the practice effects associated with the discrimination of a novel stimulus that shares important physical characteristics with both stationary lines and moving random dots. A single moving dot was chosen for several reasons. A single moving dot can be presented at the same speed and direction as a random-dot display and can be sized to match the spatial extent of each dot contained in the display. Such a single dot would differ from a random-dot pattern, however, with respect to the number of cues to the stimulus' direction. The movement of a single dot excites only a single trajectory, whereas the motion of a random-dot pattern excites many parallel paths. The single dot then is similar to the stationary line in that both provide only a single cue to the orientation/direction of the stimulus. Also, both the single-dot and the randomdot trajectories can be sized to match the length and orientation of a stationary line. The (obvious) difference between a stationary line and a single moving dot lies in the temporal aspects of each. We reasoned that if presentation duration and the length of the trajectories/stationary lines are held constant, the practice effects associated with the discrimination of these three stimuli could be more fairly compared and might serve as an index of the underlying visual mechanisms.

Several specific hypotheses can be evaluated by comparing the practice effects associated with the discrimination of single-dot, random-dot, and stationary-line stimuli. One hypothesis assumes that the different practice effects reported previously for line-orientation tasks and random-dot direction tasks were caused by a difference in the number of cues to the stimulus' orientation/ direction. If this were the case, one would expect the practice effects associated with moving-single-dot and stationary-line stimuli to be similar to each other yet consistently different from the practice effects obtained on random-dot direction discrimination task. This should be true across line/trajectory lengths (i.e., stimulus speeds), since single dots and stationary lines always provide a cue to orientation/direction at only a single location, whereas coherently moving random-dot patterns provide directional cues at multiple spatial locations. Such a finding would suggest that motion, per se, was not the cause of the different practice effects originally reported. Moreover, if the practice effects associated with stationaryline and moving-single-dot stimuli were sufficiently similar, it would be parsimonious to speculate that a common underlying mechanism subserved both perceptual processes.

A second hypothesis follows from the assumption that the differences between Vogels and Orban's (1985) study and Ball and Sekuler's (1987) study were simply due to the presence or absence of motion. If this premise were true, then the practice effects associated with movingsingle-dot and moving-random-dot stimuli should be similar to each other at each velocity yet consistently different from the practice effects obtained on the lineorientation task. Such a finding would suggest that direction and orientation processes are subserved by independent mechanisms. Consistency between the practice effects found in the single-dot and random-dot tasks would also make it parsimonious to speculate that a single motion mechanism subserves the perception of both stimulus types and that this mechanism is largely indifferent to the number of cues to the stimulus direction. 
A third possible explanation is that practice effects on these tasks depend on both the velocity of the stimulus and the number of cues to direction/orientation. A single moving dot and a stationary line could be presented such that each would excite a similar (single) path of receptors along the retinal mosaic. Although the moving dot would excite the receptors sequentially and the line would excite the receptors simultaneously, the magnitude of this difference (between sequential and simultaneous excitation) would decrease as the dot is moved faster. In the limit, the dot could be moved sufficiently fast that it would be perceived as a line. Thus, at faster speeds, one would expect similar practice effects for single-dot and stationaryline stimuli. When the single dot is presented at slower speeds, there will be a large difference between the resultant sequential excitation on the retinal mosaic and the simultaneous excitation elicited by a stationary line. If velocity matters, then there should be a difference in the practice effects associated with a slow-moving single dot and a stationary line yet similar practice effects associated with a fast single dot and a stationary line. If the number of direction cues matters, then different practice effects on the single-dot and random-dot tasks would be expected at any speed. The predictions from this third hypothesis are interesting because such results would argue against a single mechanism subserving discrimination of different stimuli moving at identical velocities (i.e., different practice effects for single- and random-dot stimuli moving at the same velocity). Furthermore, if the results are consistent with this hypothesis, it would be parsimonious to speculate that a single mechanism subserves discrimination of some stationary stimuli (e.g., stationary lines) and some fast-moving stimuli (e.g., fast single dots).

We conducted a trio of experiments to evaluate these hypotheses. In Experiment 1, practice effects for singledot direction discrimination were assessed at three velocities $(2 \% \mathrm{sec}, 10 \% \mathrm{sec}$, and $16 \% \mathrm{sec})$ and both cardinal and oblique directions of motion. Experiments 2 and 3 were carried out to allow a comparison of the practice effects associated with line-orientation and random-dot direction discrimination, respectively, by matching the trajectory length and velocities used in Experiment 1. The use of various line lengths and speeds also allowed us to determine whether the practice effects previously reported by Vogels and Orban (1985) and Ball and Sekuler (1987) were contingent on the particular stimulus parameters used in those studies.

\section{GENERAL METHOD}

\section{Observers}

Thirty-six Brown University students, ranging from 18 to 30 years of age, participated in the study. All were naive psychophysical observers with either normal or corrected-to-normal vision. Each observer was randomly assigned to an experimental condition and was paid $\$ 5$ for each of seven $1-h$ sessions.

\section{Apparatus}

All experiments were conducted on a Tektronix 608 oscilloscope with a $\mathrm{p} 31$ phosphor controlled by a Gateway $20004 \mathrm{DX} 2$ computer via a digital-to-analog converter (Computer Boards Inc., CIODAC16). While using a chinrest to provide a fixed viewing position, the participants observed the dimly presented stimuli (i.e., sufficiently dim to avoid streaking) binocularly through a circular tube that measured $57 \mathrm{~cm}$ in length and had an inner diameter of $10 \mathrm{~cm}$. In an effort to eliminate peripheral horizontal and vertical cues in the experimental room (e.g., table corners, cinder block patterns, etc.), a circular cardboard mask (34 cm in diameter with a hole in the center for the viewing tube) was affixed to the end of the viewing tube nearest the observer. For this same reason, all experiments were conducted in a darkened room, after the observers had dark adapted.

\section{Procedure}

In all experiments, the method of constant stimuli was used to assess either the observer's direction-of-motion or orientation threshold. Two stimuli, separated by a fixed temporal interstimulus interval (ISI), were presented sequentially on each trial. The observer's task was to judge the direction/orientation of the second stimulus to be either clockwise or anticlockwise (by typing "c" or "a," respectively) relative to the direction/orientation of the first stimulus. All observers were informed that the accuracy of each response was of paramount importance and that the speed of responding was irrelevant. The computer provided auditory feedback after every trial (a low tone for incorrect responses or a high tone for correct responses), and the observers established the intertrial interval by pressing the "enter" key to initiate the next trial.

Each observer practiced on just one randomly assigned standard direction/orientation for the duration of the experiment, and all trials contained at least one stimulus at that standard direction/orientation. The other stimulus in each trial was either the "standard" stimulus (on same trials) or a "test" stimulus that differed from the standard stimulus by one of two near-threshold values. On same trials, the high tone indicating a correct response was generated regardless of the observer's response. Either the standard stimulus or the test stimulus would randomly appear first on any given trial, and every block comprised 12 trials in each of the following five categories: (1) anticlockwise-large-difference, (2) anticlockwise-small-difference, (3) same, (4) clockwise-small-difference, and (5) clockwise-largedifference. The order of presentation was block randomized.

Within each stimulus condition, orthogonal directions (or orientations) at each speed (or line length) were tested. For example, in the fast cardinal condition of the motion experiments, both observers were presented with a stimulus moving at $16 \% \mathrm{sec}$, but the direction of motion was $0^{\circ}$ (rightward) for 1 observer and $90^{\circ}$ (upward) for the other. In the fast oblique condition, each of the 2 observers were required to discriminate stimuli moving at either $45^{\circ}$ (upward and rightward) or $135^{\circ}$ (upward and leftward), and so on. Similarly, one of two orthogonal orientations was discriminated by each observer assigned to any given condition in the orientation discrimination task.

The proportion of clockwise responses was tracked on each block, and probit analysis was used to determine the orientation/direction difference necessary to change performance levels from $50 \%$ to $75 \%$. The experimenter reviewed the performance summary with the observer after every block and selected the stimulus set for the next block accordingly. For example, when the observer consistently discriminated $8^{\circ}$ and $4^{\circ}$ test stimuli, the stimulus set comprising $4^{\circ}$ and $2^{\circ}$ stimuli would be presented until the observer's performance warranted use of the $2^{\circ}$ and $1^{\circ}$ stimuli, and so on. The least discriminable stimulus set contained tests of $0.25^{\circ}$ and $0.125^{\circ}$.

Thirty practice trials preceded the first block of the first session to ensure that the task was understood before data collection. Data were collected in seven 1 -h sessions, with each daily session consisting of ten 60 -trial blocks. The seven sessions were conducted over a 1 - to 2-week period, with the constraint that no two sessions could be conducted on the same day. In total, each of the 36 observers made 4,200 clockwise/anticlockwise judgments. Two criteria were used to ensure the accuracy of each threshold measurement. First, 
the chi-square statistic (which indicates whether the data are well characterized by a cumulative normal curve) for each block had to be less than the critical value at the .01 alpha level; second, the standard error of the threshold ( $S E T$ ) had to be less than the value of the threshold itself.

To determine the effect of practice for each observer, the 10 thresholds obtained in each daily session were averaged, thereby resulting in 7 thresholds (each based on 600 trials) for every observer. Linear and nonlinear trends in the data were assessed via productmoment correlations between practice and threshold and between log-practice and log-threshold, respectively. While the correlation coefficients from the linear and nonlinear analyses were quite similar, the nonlinear functions generally provided a better fit to the data. For brevity, only the correlations associated with the nonlinear analyses will be presented. Because perceptual learning was operationalized as a decrease in visual threshold caused by the repetition of a visual task, correlation coefficients ranging from -.75 to -1.0 were taken as evidence of significant perceptual learning $\left[r_{\text {crit }}(5)=-.75, \alpha=.05\right]$. To assess the time course of learning, product-moment correlations were conducted on the log of the 10 thresholds obtained from each observer in each daily session. Correlation coefficients of -.63 or beyond $\left[r_{\text {crit }}(8)=-.63, \alpha=.05\right]$ would indicate that significant learning transpired within a daily session. For observers who demonstrate an overall threshold reduction, a failure to find these within-session threshold declines would imply that learning occurred gradually over the course of many sessions.

The effect of the various stimuli on threshold reduction was evaluated in two ways. First, separate within-subjects analyses of variance (ANOVAs) were conducted for each stimulus condition, with $\log$-practice (i.e., log-trial number) as the within-subjects factor and $\log$-threshold as the dependent measure. Linear trend analyses were then calculated on the log-practice by log-threshold values - a procedure equivalent to fitting a power function to the data. Significant $F_{\text {linear }}$ values would indicate that practice significantly affected thresholds but would provide no information about the magnitude of threshold reduction per se. For this reason, a second analysis was performed by calculating the slope of the power function fit to the mean scores across all observers in each stimulus condition. To determine whether the slopes associated with any two stimulus conditions differed from each other, the difference between mean thresholds obtained in the two conditions was calculated for each of the seven daily sessions, and a power function was fit to the resultant set of difference scores. A significant between-group difference in slope would be indicated by a power function having a correlation coefficient stronger than \pm .75 . We feel that these two measuresthe ANOVA-based trend analysis, which indicates whether practice significantly affected thresholds in a particular stimulus condition, and the comparison of slopes, which indicates whether the rate of learning differed in any pair of conditions--provide a more complete assessment of perceptual learning than would either measure alone.

\section{EXPERIMENT 1 Single-Dot Direction Discrimination}

\section{Method}

Twelve observers were asked to identify a single dot's direction of motion. Two observers were randomly assigned to each of six stimulus conditions characterized by a particular speed and direction of motion. The six stimulus conditions consisted of three speeds $\left(2 \% \mathrm{sec}, 10^{\circ} / \mathrm{sec}\right.$, and $\left.16^{\circ} / \mathrm{sec}\right)$ and two directions of motion (cardinal and oblique). The two single-dot presentations were separated by a $200-\mathrm{msec}$ ISI. For all three stimulus speeds, the distance between dot positions on consecutive frames was $1.62^{\prime}$ of are. In the $16 \%$ sec condition, each single-dot presentation comprised 298 frames, and each frame was displayed for $1.68 \mathrm{msec}$, thereby creating a dot that moved $8 \mathrm{~cm}\left(8^{\circ}\right)$ in $500.64 \mathrm{msec}$. In the $10^{\circ} / \mathrm{sec}$ con- dition, 186 frames were each displayed for $2.69 \mathrm{msec}$, resulting in a single dot moving across a $5-\mathrm{cm}\left(5^{\circ}\right)$ trajectory in $500.34 \mathrm{msec}$. In the $2 \% / \mathrm{sec}$, the stimulus consisted of thirty-seven $3.6-\mathrm{msec}$ frames and traversed a $1-\mathrm{cm}\left(1^{\circ}\right)$ path in $503.2 \mathrm{msec}$.

To ensure that the observers could not reliably make the discrimination by comparing the starting or ending positions of the two single-dot presentations, the initial position of the stimulus was randomly determined on each trial. The random spatial offset was a constant fraction ( $11 \%$ ) of the trajectory length: $0.87,0.54$, and $0.11 \mathrm{~cm}$ for the fast, medium, and slow speeds, respectively. For all stimulus conditions, the spatial offset was sufficiently large to preclude the effective use of starting- and ending-point cues and was sufficiently small to keep the stimulus near the center of the screen. Because the stimulus was centered, no fixation point was provided, since its presence may have given a spatial cue to the stimulus' direction.

Of the 840 trial blocks ( 12 observers $x 70$ blocks per observer) in Experiment 1, there were only 5 blocks with chi-square values that failed to meet the above criterion, and no more than two of these were from a single observer. There was only one block with an unacceptably high $S E T$. Additional trial blocks were conducted so as not to compromise statistical power.

\section{Results}

The effect on individuals. Of the 12 observers in Experiment 1,3 demonstrated significant perceptual learning, 6 showed nonsignificant declines in threshold, and 3 demonstrated a nonsignificant positive correlation between practice and threshold. An analysis of the 10 thresholds obtained in each daily session indicated no consistent evidence for fast within-session learning for any observer.

The overall effects of direction and speed. The effect of practice on each of the two direction conditions was first evaluated with the separate within-subject ANOVA trend analyses described in the General Method section. Combining across all speed conditions, significant practice effects were found in both the cardinal $[F(1,30)=$ $7.67, p<.01]$ and the oblique $[F(1,30)=18.57, p<.01]$ conditions. The comparison of slopes revealed comparable rates of threshold reduction in the cardinal and oblique conditions after averaging across speeds $[r(5)=.69$, n.s. $]$.

Using the same procedure, correlation coefficients were also determined for the three possible pairwise speeddifference scores $\left(2 \% / \mathrm{sec}\right.$ vs. $10^{\circ} / \mathrm{sec}, 2^{\circ} / \mathrm{sec}$ vs. $16^{\circ} / \mathrm{sec}$, and $10 \% \mathrm{sec}$ vs. $16 \% \mathrm{sec}$ ). The analysis revealed that the rate of threshold reduction observed at the slowest speed significantly exceeded the rate of threshold reduction at each of the two faster speeds $[2 \% \mathrm{sec}$ vs. $10 \% \mathrm{sec}, r(5)=-.958$, $p<.01 ; 2 \%$ sec vs. $\left.16^{\circ} / \mathrm{sec}, r(5)=-.964, p<.01\right]$. There was no significant difference between the two faster speeds with respect to the rate of threshold reduction $\left[10^{\circ} / \mathrm{sec}\right.$ vs. $16^{\circ} / \mathrm{sec}, r(5)=.041$, n.s.]. Because the results at the two faster speeds were so similar to each other, the thresholds from these conditions were aggregated to permit a single slow versus fast comparison. Threshold reduction occurred at a significantly greater rate in the slow condition than in the fast condition $[r(5)=-.964, p<.01]$. Indeed, while practice had a significant effect in the slow condition $[F(1,18)=34.55, p<.01]$, thresholds were unaffected by practice in the fast condition $[F(1,42)=$ 0.20, n.s.]. 
The effect of stimulus velocity. The failure to find an overall effect for direction may have been caused by an interaction of speed and direction. To investigate this possibility and to more fully evaluate the effect of stimulus velocity, separate analyses were conducted on the daily thresholds obtained from the observers assigned to each of the four velocity conditions. There was significant threshold reduction for the discrimination of both cardinal $[F(1,6)=29.62, p<.01]$ and oblique directions of motion $[F(1,6)=14.63, p<.01]$ when the stimulus moved at $2 \% \mathrm{sec}$. At the (combined) faster speeds, however, perceptual learning was evident in the oblique condition $[F(1,18)=7.46, p<.05]$, but not in the cardinal condition $[F(1,18)=0.006$, n.s. $]$. Mean thresholds for the slow and fast conditions are plotted as a function of practice (i.e., the number of trials) in Figures 1 and 2, respectively.

The slope of each function is given by the exponent associated with the $x$ value of the power equation shown in the legend. These two figures also reveal a substantial degree of overlap between oblique and cardinal thresholds for slow stimuli, but not for the faster stimuli. To determine whether differential learning occurred between the two direction conditions at each speed, the slopes obtained in the cardinal and oblique conditions were compared separately for the slow and fast speeds. As can be seen in Figure 3, the differences between oblique and cardinal thresholds at the slow speed did not follow any discernible pattern $[r(5)=+.066$, n.s.]. The same was not true at the faster speeds. Threshold reduction occurred at a significantly faster rate in the oblique condition than in the cardinal condition $[r(5)=-.828, p<.05]$.

\section{Discussion}

The trend analyses conducted on the various stimulus conditions suggest that the pattern of threshold reduction

\section{Slow Single Dot $(2 \% / \mathrm{sec})$}

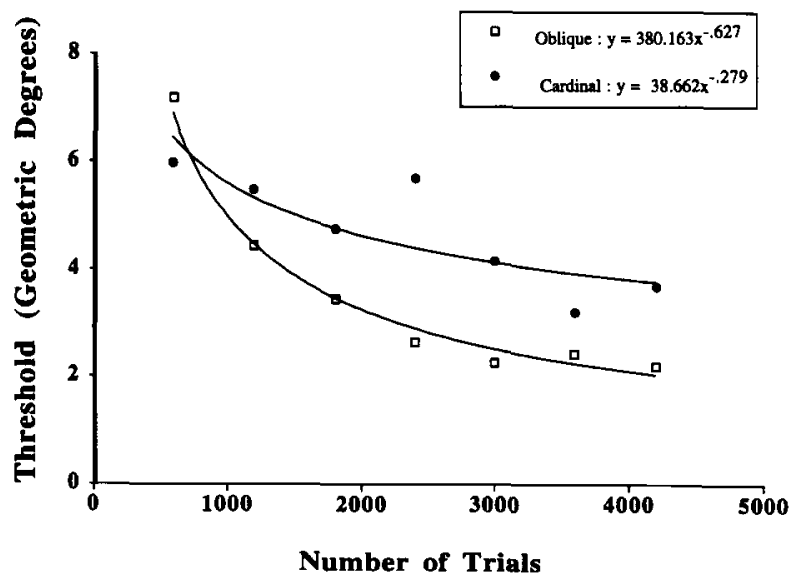

Figure 1. Slow single-dot data. The change in threshold, as a function of practice, is shown for both cardinal and oblique stimuli in the slow single-dot condition $(2 \% \mathrm{sec})$. Each point represents the average threshold of observers in the slow cardinal and slow oblique conditions. Cardinal and oblique thresholds overlapped during training, and both were reduced significantly by practice.
Fast Single Dot $(10 \% / \mathrm{sec}$ and $16 \% \mathrm{sec})$

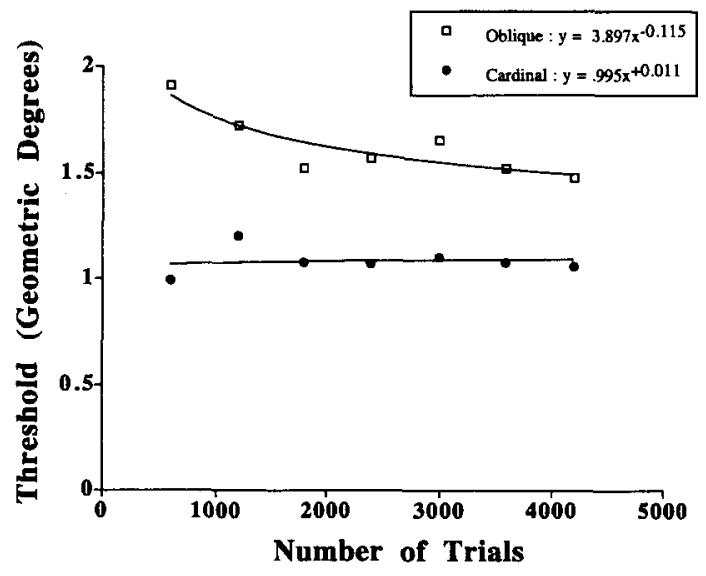

Figure 2. Fast single-dot data. The change in threshold, as a function of practice, is shown for both cardinal and oblique stimuli in the (combined) fast single-dot condition $(10 \%$ sec and $16 \%$ sec). Because practice had comparable effects $10 \% / \mathrm{sec}$ and $16 \% \mathrm{sec}$, thresholds from these two speed conditions were combined. Practice significantly affected discrimination at only the oblique directions, and there was no overlap between cardinal and oblique thresholds throughout the training period.

for the observers in Experiment 1 depends on the effect of stimulus velocity. This conclusion follows from the observation that significant threshold reduction occurred at both cardinal and oblique directions when the stimulus moved slowly, but only at the oblique directions when the speed was $10 \% \mathrm{sec}$ or $16 \% \mathrm{sec}$. The comparison of slopes lends further support for this conclusion. The rates of threshold reduction in the cardinal and oblique conditions were comparable at the slow speed; however, at faster speeds, threshold reduction occurred at a significantly greater rate in the oblique condition than in the cardinal condition. What is more, throughout training there was overlap between oblique and cardinal thresholds when the stimulus moved at only $2 \% \mathrm{sec}$, but no overlap at any point in training when speed was $10 \% \mathrm{sec}$ or $16 \% \mathrm{sec}$.

Some comparisons between the results from Experiment 1 of the present study and those reported previously for the random-dot task (Ball \& Sekuler, 1987) can be made, since both studies had at least one condition in which the stimulus moved at $10 \% \mathrm{sec}$. Interestingly, the practice effects associated with these two types of stimuli are very different despite the fact that both moved at the same velocity for the same amount of time. Significant learning occurred only at the oblique directions in the single-dot task, but at both directions in the random-dot task. Also, in the single-dot case, the rate of learning was significantly greater at the oblique direction than at the cardinal direction, yet this difference did not occur in the random-dot case. Finally, Ball and Sekuler (1987) reported that, with practice, performance in the oblique condition was better than the initial performance in the cardinal condition. We found no overlap in oblique and cardinal thresholds at any point in training for our $10 \% \mathrm{sec}$ 


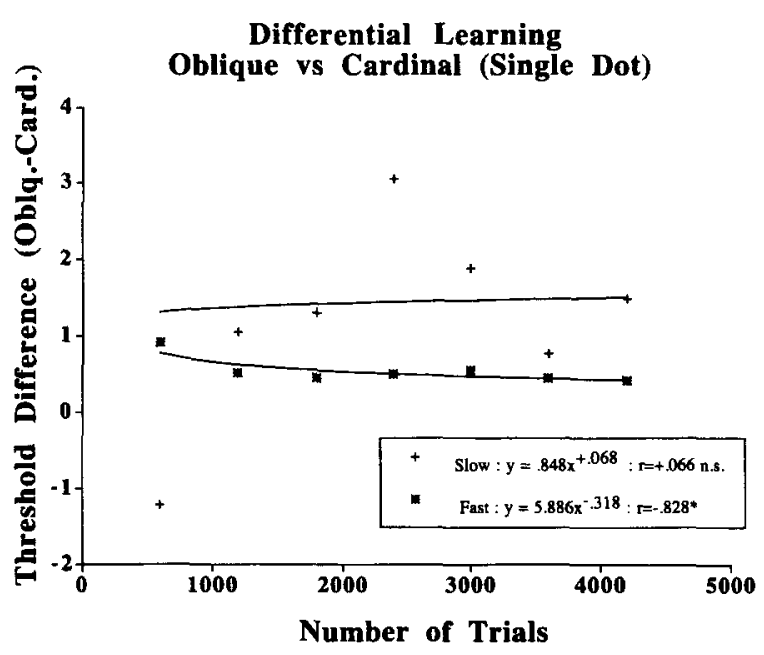

Figure 3. A comparison of cardinal and oblique directions (single-dot case). The slopes associated with oblique and cardinal directions are compared in order to determine whether the rate of perceptual learning differed in a direction-specific manner. The difference between mean daily oblique thresholds and mean daily cardinal thresholds is shown separately for the slow and the (combined) fast single-dot conditions. The poor fit associated with the slow speed suggests comparable rates of threshold reduction in the slow oblique and slow cardinal conditions. By contrast, the significant fit associated with the fast speeds suggests that the rate of threshold reduction in the fast oblique condition was significantly greater than that in the fast cardinal condition.

single-dot stimulus. All of these differences also hold true if the comparison is made between Ball and Sekuler's random-dot results and our $16 \%$ sec single-dot condition. Indeed, we find no differences between the practice effects associated with our $10 \% \mathrm{sec}$ and $16 \% \mathrm{sec}$ single-dot stimuli.

The differences between our single-dot results and Ball and Sekuler's (1987) random-dot results suggest that different mechanisms may be subserving direction discrimination of the stimuli. Because slightly different procedures were used in the two experiments, however, the comparisons above might not be entirely fair. Specifically, the observers in our single-dot experiment were asked to identify differences in direction of motion (i.e., clockwisel anticlockwise judgments), whereas Ball and Sekuler (1987) simply asked their observers to detect directional differences in the random-dot patterns (i.e., sameldifferent judgments). To ensure that this procedural difference was not the source of the discrepant results and to allow a more complete assessment of the three hypotheses set forth in our introduction, we conducted two additional identification (as opposed to detection) experiments using stationary-line and random-dot stimuli. We chose to use new subjects in each of these remaining experiments because a failure to find significant perceptual learning in the observers who had participated in Experiment 1 would be difficult to interpret. Carryover effects could not be distinguished from a genuine lack of plasticity.

\section{EXPERIMENT 2 Line-Orientation Discrimination}

\section{Method}

In Experiment 2, the observers practiced identifying differences in the orientation of sequentially presented lines. To avoid possible carryover effects, 12 new naive observers were used. Two observers were randomly assigned to each of six stimulus conditions characterized by a particular line length and orientation. The six stimulus conditions consisted of two orientations (cardinal and oblique) and three line lengths. Line lengths of $1^{\circ}, 5^{\circ}$, and $8^{\circ}$ were used to match the lengths of the trajectories traversed by the single-dot stimulus in Experiment 1. Within each stimulus condition, orthogonal orientations were tested in the manner described in the General Method section.

Experiment 2 was designed to parallel the single-dot paradigm, but we found that an ISI of $200 \mathrm{msec}$ resulted in apparent motion between the lines in the two intervals. Consequently, the two 500msec stimulus presentations in Experiment 2 were separated by a $1,000-\mathrm{msec}$ ISI. This $1: 2$ ratio of stimulus duration to ISI is consistent with that used by Vogels and Orban (1985), who employed a 1,200-msec ISI between 600-msec line presentations.

As in Experiment 1, a random spatial offset was generated on each trial to prevent the observers from reliably discriminating differences in the location of corresponding points on the two stimuli. The parameters that characterized the spatial offset for the $1^{\circ}, 5^{\circ}$, and $8^{\circ}$ lines were identical to those used for the $2 \% \mathrm{sec}, 10^{\circ} / \mathrm{sec}$, and $16 \%$ sec conditions, respectively, in Experiment 1.

The performance of each observer was assessed by probit analysis on a block-by-block basis. The accuracy criteria for Experiment 2 were the same as those used earlier. All of the 840 blocks of trials were characterized by SETs that satisfied the requisite accuracy level, and there was only one block with an unacceptably high chisquare statistic. The threshold for that block was discarded, and another 60-trial block was taken.

\section{Results}

The effect on individuals. The effect of practice on orientation discrimination thresholds was measured using all of the same calculations described in the General Method section. Of the 12 observers in Experiment 2, 4 demonstrated significant perceptual learning, 6 showed nonsignificant declines in threshold, 1 demonstrated a significant threshold increase, and 1 showed a nonsignificant threshold increase. An analysis of the 10 thresholds obtained in each daily session indicated no consistent evidence for fast within-session learning for any observer.

The overall effects of orientation and line length. Combining across all line lengths, significant practice effects occurred at the oblique orientations $[F(1,30)=$ $14.64, p<.01]$, but not at the cardinal orientations where thresholds increased nonsignificantly $[F(1,30)=0.20$, n.s.]. Because threshold reduction at the oblique orientations was significantly different from zero, whereas thresholds actually increased at the cardinal orientations, the statistical procedure used in Experiment 1 to infer differential rates of learning is unnecessary. It is intuitively obvious that the rates of perceptual learning differed in an orientation-specific manner.

Differential learning was assessed in the three pairwise length conditions ( 1 vs. $5 \mathrm{~cm}, 1$ vs. $8 \mathrm{~cm}$, and 5 vs. $8 \mathrm{~cm}$ ) after averaging across orientations. The analysis revealed 
that the rate of threshold reduction observed at the shortest length significantly exceeded the rate of threshold reduction at each of the two longer lengths $[1 \mathrm{vs} .5 \mathrm{~cm}$, $r(5)=-.812, p<.05 ; 1$ vs. $8 \mathrm{~cm}, r(5)=-.854, p<.05]$. Differences between the $8-\mathrm{cm}$ condition and the $5-\mathrm{cm}$ condition produced a mixture of positive and negative scores that could not be analyzed the same way (i.e., with a power function). However, a linear analysis revealed that the difference between the rates of threshold reduction at these two longer lengths was not significant [5 vs. $8 \mathrm{~cm}$, $r_{\text {linear }}(5)=+.528$, n.s.]. Because the results at the two longer lengths did not differ from each other, the thresholds from these conditions were aggregated to permit a single short versus (combined) long comparison. Analysis of the difference between the short and long conditions indicated that the rate of perceptual learning was significantly greater at the short line length $[r(5)=-.855$, $p<.05]$. Thresholds also declined significantly with practice in the short condition $[F(1,18)=6.51, p<.05]$, but not in the long condition $[F(1,42)=+.0004$, n.s.].

The combined effect of length and orientation. The failure to find perceptual learning in the longer conditions may have been caused by an interaction of orientation and line length. To evaluate this possibility, the daily thresholds obtained from the observers assigned to each of the four stimulus conditions ( 2 orientations at short and long line lengths) were evaluated separately. Practice had a nonsignificant effect on thresholds in the shortline condition. This was true at both the cardinal $[F(1,6)=$ 1.82 , n.s. $]$ and the oblique $[F(1,6)=4.15$, n.s. $]$ orientations. At the longer line lengths, however, thresholds declined significantly in the oblique condition $[F(1,18)=$ $8.07, p<.05$ ] but increased nonsignificantly in the cardinal condition $[F(1,18)=2.79$, n.s. $]$. Mean thresholds for the short and long conditions are plotted as a function of practice in Figures 4 and 5, respectively. Inspection of these two figures also reveals that there is an overlap between oblique and cardinal thresholds in the short-line condition, but not in the longer line condition. As can be seen in Figure 6, the nonsignificant correlation coefficient associated with the oblique-cardinal comparison in the short condition indicates that learning at the two orientations occurred at comparable rates $[r(5)=-.121$, n.s.]. However, at the longer lengths, learning occurred at a significantly greater rate in the oblique condition than in the cardinal condition $[r(5)=-.846, p<.05]$.

\section{Discussion}

The pattern of threshold reduction observed in the longline conditions is consistent with that reported previously by Vogels and Orban (1985). At the longer line lengths, there was no overlap between oblique and cardinal thresholds at any point in training, and a significant practice effect was observed only in the oblique condition. In both the present experiment and Vogels and Orban's (1985) study, there was significantly greater learning in the oblique condition than in the cardinal condition. The results from the longer line conditions of the present experiment there-

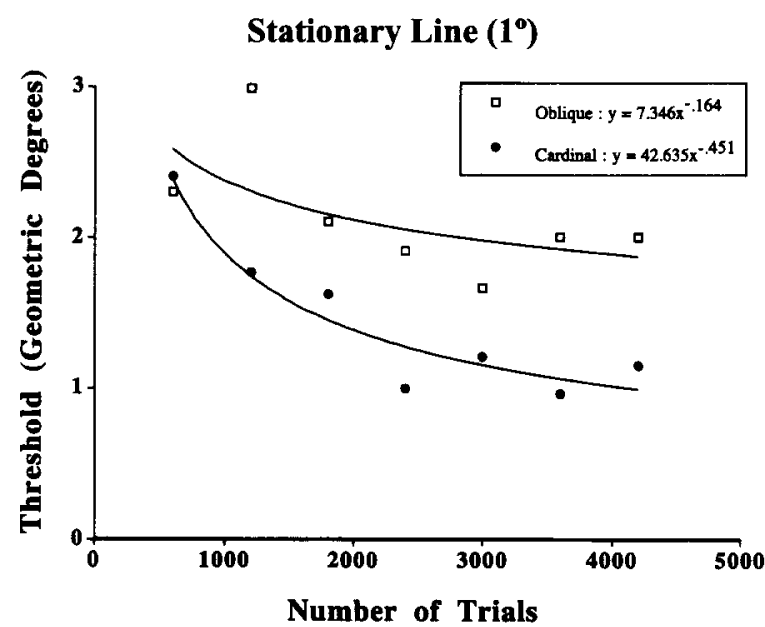

Figure 4. Short line-orientation data. The effect of practice is shown separately for cardinal and oblique thresholds in the short line-orientation condition. The stimuli subtended $1^{\circ}$ of visual angle. Each point represents the average threshold of the observers in the short cardinal and short oblique conditions. Cardinal and oblique thresholds overlapped during training, and neither was reduced significantly by practice.

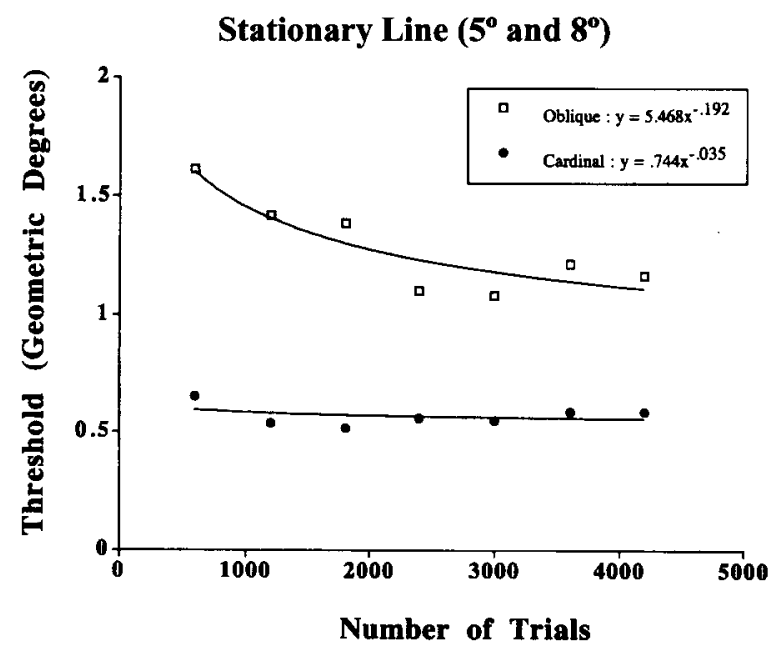

Figure 5. Long line-orientation data. The effect of practice is shown separately for cardinal and oblique thresholds in the (combined) long line-orientation condition. Because practice had comparable effects on the discrimination of $5^{\circ}$ and $8^{\circ}$ stimuli, thresholds from these two length conditions were combined. Practice significantly affected discrimination at only the oblique orientation, and there was no overlap between cardinal and oblique thresholds at any point in training.

fore confirm and extend those reported earlier by Vogels and Orban (1985) for $15^{\circ}$ stimuli to $8^{\circ}$ and $5^{\circ}$ stimuli.

Another pattern observed in Experiment 2 is consistent with the claim that the oblique effect decreases with decreasing line length (Vandenbussche et al., 1983; Vogels et al., 1984): There was no evidence for perceptual learning at either orientation when the stimulus was only 


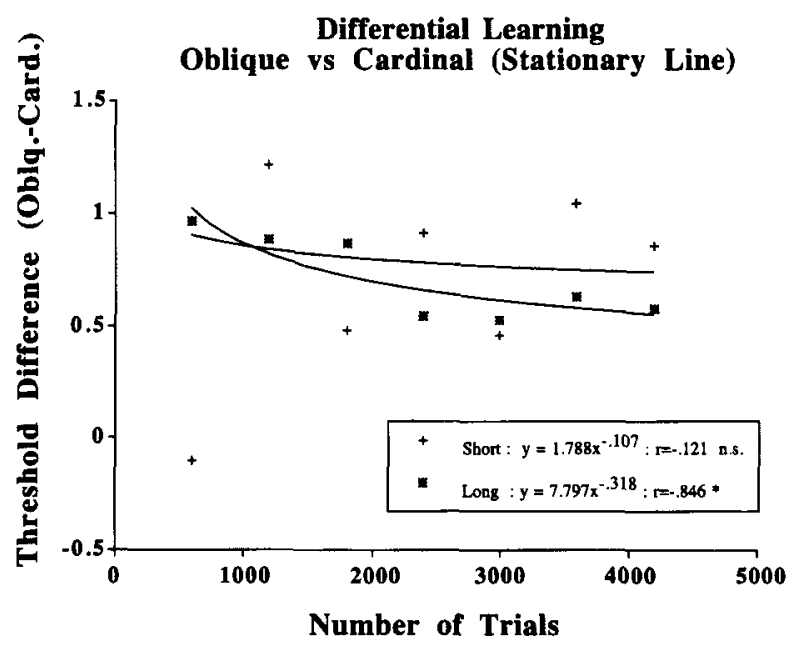

Figure 6. A comparison of oblique and cardinal orientations (line-orientation case). The slopes associated with oblique and cardinal orientations are compared in order to determine whether the rate of perceptual learning differed in an orientation-specific manner. The difference between mean daily oblique thresholds and mean daily cardinal thresholds is shown separately for the short and the (combined) long single-dot conditions. The poor fit associated with the short oblique and short cardinal conditions suggests comparable rates of threshold reduction at the two orientations. By contrast, the significant fit associated with the longer line lengths suggests that the rate of threshold reduction in the long oblique condition was significantly greater than that in the long cardinal condition.

$1 \mathrm{~cm}$ in length. Furthermore, unlike the results observed at the longer line lengths, there was no evidence for differential learning between the two orientations in the short condition, and there was some overlap between oblique and cardinal thresholds. The effect of line length may therefore explain why there was a failure to detect a significant main effect of orientation. The large difference between the learning effects in the oblique and cardinal conditions at longer line lengths may have been masked by the results from the short-line condition. The failure to find significant perceptual learning in the short oblique condition also implies that the orientationspecific learning reported by Vogels and Orban (1985) may be contingent on line length.

The difference in practice effects observed in the shortand long-length conditions of Experiment 2 can be compared with the differences in the slow and faster conditions of Experiment 1 to evaluate the first hypothesis set forth in our introduction. That hypothesis held that the difference in the practice effects reported in the earlier randomdot (Ball \& Sekuler, 1987) and line-orientation (Vogels \& Orban, 1985) studies was caused solely by a difference in the number of cues to direction/orientation. One prediction that follows from this hypothesis is that the practice effects associated with stationary-line and movingsingle-dot stimuli should be comparable across line/ trajectory lengths (speeds), since both stimuli always provide the same number of cues to direction/orientation (i.e., one). The data from Experiments 1 and 2 argue against this. The length of the trajectory in our slow single-dot task matched the length of the stationary lines in our short orientation task, yet very different patterns of threshold reduction were evident in the two tasks. Thresholds dropped significantly $(p<.01)$ on the slow single-dot direction task whether the stimuli moved cardinally or obliquely. By contrast, significant threshold reduction occurred in neither the short oblique line-orientation condition nor the short cardinal line-orientation condition. The differences between the results from Experiments 1 and 2 suggest that the velocity of the stimulus does affect the pattern of threshold reduction and that the different practice effects reported earlier by Vogels and Orban (1985) and Ball and Sekuler (1987) cannot be attributed only to differences in the number of cues provided by stationary-line and moving-random-dot stimuli.

To evaluate the second and third hypotheses, it was necessary to conduct a final experiment in which the observers were asked to identify the direction of random-dot stimuli. Because the speed/trajectory lengths of our random-dot patterns were matched to those of Experiments 1 and 2, direct comparisons could be made between the practice effects associated with these various stimuli. The use of multiple speeds also allowed us to assess whether the results originally reported by Ball and Sekuler (1987) were contingent on the specific speed at which their stimuli moved $(10 \% \mathrm{sec})$.

\section{EXPERIMENT 3 Random-Dot Motion Discrimination}

\section{Method}

The observers in Experiment 3 were required to discriminate the direction of moving random dots. The various stimulus speeds and directions and the random assignment procedures were identical to those of Experiment 1. Twelve new naive observers were used in Experiment 3 to avoid possible carryover effects.

The stimuli for this experiment were 256 spatially random dots traveling in parallel paths across a $10 \times 10 \mathrm{~cm}$ square patch on the oscilloscope. Each dot disappeared after reaching the boundary of the square patch and was immediately replaced by another dot on the opposite side. When seen through the $10-\mathrm{cm}$ circular viewing tube, the mean dot density was 2.5 dots per square $\mathrm{cm}$. For all three stimulus speeds, 25 frames were presented (each for a duration of $20 \mathrm{msec}$ ) in $500 \mathrm{msec}$. This frame rate $(50 \mathrm{~Hz})$ exceeded the minimum necessary to achieve the percept of smooth motion (Ball \& Sekuler, 1979). The ISI was $200 \mathrm{msec}$ in all conditions. The distance between dot positions on consecutive frames was dependent on the stimulus speed; the spatial hop sizes were $19.02 \mathrm{arc}$ min, $12 \mathrm{arc}$ min, and 2.4 arc $\mathrm{min}$, in the $16 \% \mathrm{sec}, 10 \% \mathrm{sec}$, and $2 \% \mathrm{sec}$ conditions, respectively.

A new set of random dots was generated for each block of 60 trials. To prevent the observers from reliably using the relative positions of identifiable dot clusters within a trial block, different sequences of frames were used in each stimulus presentation. This was accomplished by presenting only 25 of any 33 consecutive frames stored in the computer memory. For example, if the first stimulus was to be produced by Frames 1-25, the second stimulus could be produced by Frames $8-33$. In this way, corresponding dot clusters would be spatially offset even on same trials.

Random-dot direction discrimination thresholds were determined in the manner described in the General Method section. The accuracy of each measurement was ensured by the chi-square and SET criteria previously stated. Only 5 of the 840 trial blocks had to be dis- 
carded ( 3 with unacceptable $S E T \mathrm{~s}$, and 2 with unacceptable chisquare values), and each of these was produced by a different observer. An additional trial block was conducted in every case to retain statistical power.

\section{Results}

The effect on individuals. The effect of practice on direction discrimination thresholds was measured using calculations described in the General Method section. All 12 observers in Experiment 3 demonstrated a decline in threshold over the course of training; however, the practice effects were statistically significant for only 3 observers. An analysis of the 10 thresholds obtained in each daily session indicated no consistent evidence for fast within-session learning for 11 of the 12 observers. One observer demonstrated significant within-session learning on five of the seven daily sessions.

The overall effects of direction and speed. Combining across the three speed conditions, significant practice effects were evident in both the cardinal condition $[F(1,30)=4.84, p<.05]$ and the oblique condition $[F(1,30)=22.67, p<.01]$. The slopes of the daily means in each of the two direction conditions were also compared in order to assess whether the rate of threshold reduction differed in a direction-specific manner. This analysis indicated that the rates of threshold reduction in the cardinal and oblique conditions were statistically indistinguishable from each other $[r(5)=-.215$, n.s.].

Using the same procedure, differential learning was assessed in the three pairwise speed conditions $(2 \% \mathrm{sec}$ vs. $10 \% \mathrm{sec}, 2 \% \mathrm{sec}$ vs. $16^{\circ} / \mathrm{sec}, 10 \% \mathrm{sec}$ vs. $16 \% \mathrm{sec}$ ) after averaging across directions. No significant differences were found between any of the speed conditions $[2 \% \mathrm{sec}$ vs. $10 \% / \mathrm{sec}, r(5)=-.351$, n.s.; $2 \% / \mathrm{sec}$ vs. $16 \% \mathrm{sec}, r(5)=$ -.143 , n.s.; $10 \% \mathrm{sec}$ vs. $16 \% \mathrm{sec}, r(5)=-.037$, n.s.]. To match the analyses conducted on the first two experiments, the results from the two faster speeds were aggregated to permit a single fast versus slow comparison. A comparison between the thresholds obtained at the slow and fast speeds indicated that the rate of perceptual learning did not differ in these two speed conditions $[r(5)=-.251, \mathrm{n} . \mathrm{s}$.]. Also, thresholds did not decline significantly with practice in either the slow condition $[F(1,18)=2.79$, n.s. $]$ or the combined fast conditions $[F(1,18)=.59$, n.s. $]$ when the results were aggregated across oblique and cardinal directions.

The effect of velocity. To determine whether the failure to find main effects for speed and direction was caused by a significant interaction, separate analyses were conducted on the daily thresholds obtained from the observers assigned to each of the four stimulus conditions (two directions, two speeds). The results from the two faster speeds were combined to match the analyses conducted in the first two experiments. At the slow speed, nonsignificant practice effects were obtained in the oblique condition $[F(1,6)=2.81$, n.s. $]$ and in the cardinal condition $[F(1,6)=0.51$, n.s.]. At the faster speeds, however, significant practice effects occurred in both the oblique condition $[F(1,18)=20.15, p<.01]$ and the car- dinal condition $[F(1,18)=7.78, p<.05]$. Mean thresholds for the slow and fast conditions are shown as a function of trial number in Figures 7 and 8, respectively.

The figures reveal that there was at least some overlap between oblique and cardinal thresholds at both slow and fast speeds. The nonsignificant correlation coefficients in Figure 9 indicate that differential learning rates were not found between the two direction conditions at either

Slow Random Dots (2\%/sec)

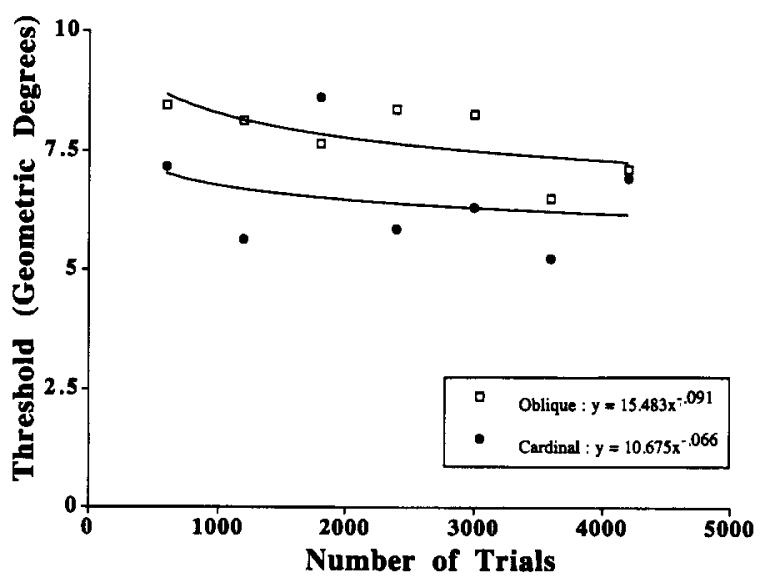

Figure 7. Slow random-dot data. The change in threshold, as a function of practice, is shown for both cardinal and oblique stimuli in the slow random-dot condition $(2 \% \mathrm{sec})$. Each point represents the average threshold of the observers in the slow cardinal and slow oblique conditions. Cardinal and oblique thresholds overlapped during training, but neither was reduced significantly by practice.

Fast Random Dots $\left(10^{\circ} / \mathrm{sec}\right.$ and $16 \%$ sec)

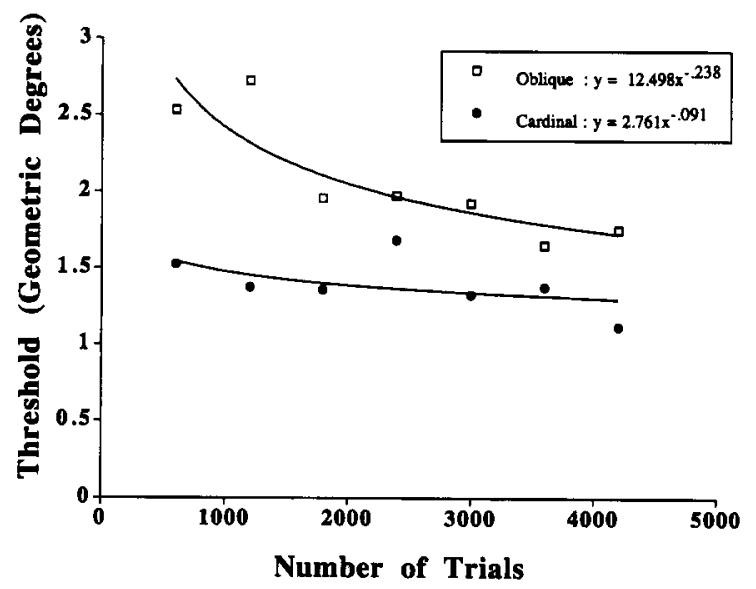

Figure 8. Fast random-dot data. The change in threshold, as a function of practice, is shown for both cardinal and oblique stimuli in the (combined) fast random-dot condition $(10 \% / \mathrm{sec}$ and $16 \%$ sec). Thresholds from the $10 \%$ sec and $16 \%$ sec conditions were combined to match the analyses conducted in the single-dot and line-orientation experiments of the present study. Practice on the fast random-dot task significantly affected discrimination at both directions, and there was some overlap between cardinal and oblique thresholds. 
the slow speed $[r(5)=-.406$, n.s.] or the faster speeds $[r(5)=-.662$, n.s. $]$.

\section{Discussion}

The results from the fast conditions in Experiment 3 of the present study are very similar to those reported earlier by Ball and Sekuler (1987) in several ways. First, unlike the pattern of threshold reduction observed in the long-line condition of Experiment 2, there was some overlap between the mean daily thresholds of the observers in the oblique conditions and the mean daily thresholds of the observers in the cardinal conditions. As Ball and Sekuler (1987) found, the oblique effect was still present at the conclusion of training; the final thresholds in the cardinal conditions were less than the final thresholds observed in the oblique conditions. This was particularly evident at the combined speeds of $10^{\circ} / \mathrm{sec}$ and $16 \% \mathrm{sec}$ (Ball \& Sekuler's, 1987, stimuli moved at $10 \% \mathrm{sec}$ ). Other similarities between the two studies pertain to the effect of practice and the rate of perceptual learning. When the stimulus moved at $10 \% \mathrm{sec}$ (or faster) in both studies, significant perceptual learning occurred in both the cardinal direction and the oblique direction, and the rate at which perceptual learning transpired was comparable in the two direction conditions. This pattern of threshold reduction was not observed in Experiment 2, where the rate of threshold reduction was significantly greater in the long-oblique-line conditions than in the long-cardinalline conditions.

One important difference between the two random-dot studies is that our Experiment 3 assessed the effect of speed on threshold reduction. Although stimulus speed was manipulated in one of the experiments in the previ-

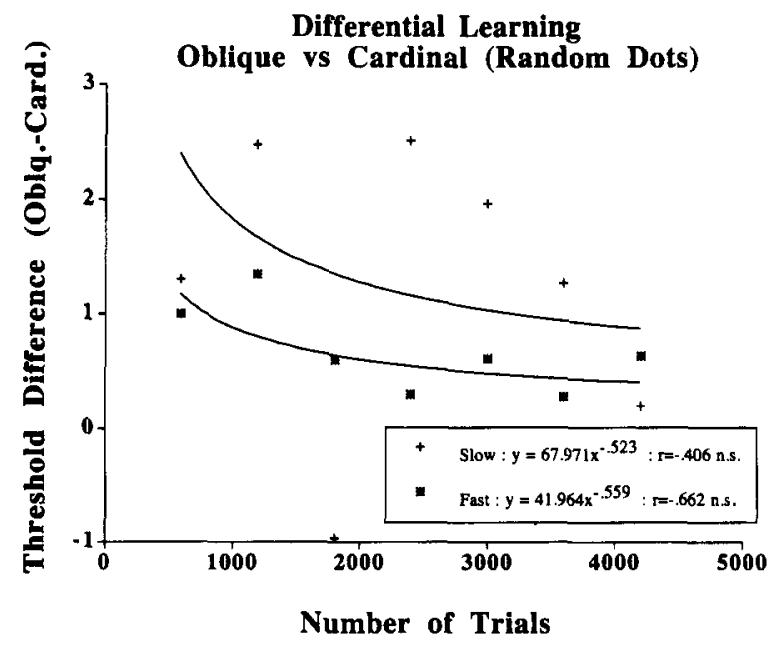

Figure 9. A comparison of oblique and cardinal directions (random-dot case). The slopes associated with oblique and cardinal directions are compared in order to determine whether the rate of perceptual learning differed in a direction-specific manner. The difference between mean daily oblique thresholds and mean daily cardinal thresholds is shown separately for the slow and the (combined) fast random-dot conditions. The poor fit suggests that learning occurred at comparable rates in the oblique and cardinal conditions regardless of speed. ous study (Ball \& Sekuler, 1987), only initial thresholds were measured, not the change in thresholds with practice as a function of speed. While both investigations found that performance was much worse overall at very slow speeds (i.e., $2^{\%} / \mathrm{sec}$ ), the results from Experiment 3 of the present study suggest that practice-based improvements in random-dot direction discrimination may depend on speed. Thresholds did not decline significantly in the present study when the random-dot stimuli moved at only $2 \% \mathrm{sec}$. This was true for both cardinal and oblique directions of motion. The significant practice effects reported previously for random-dot direction discrimination tasks (Ball \& Sekuler, 1987) may therefore be contingent on a particular speed $(10 \% \mathrm{sec})$.

The effect of velocity on thresholds in Experiments 1 and 3 can be evaluated to determine the validity of the second hypothesis stated in the introduction - the differences in the practice effects reported previously by Vogels and Orban (1985) and Ball and Sekuler (1987) were simply due to the presence or absence of motion. A prediction from this hypothesis is that the practice effects associated with two different moving stimuli should not depend on the number of cues to direction that each stimulus contains, so long as the two stimuli move at the same velocity. The data from Experiments 1 and 3 are not consistent with this prediction. At the slow speed $(2 \% / \mathrm{sec})$, significant threshold reduction occurred in both oblique and cardinal directions on the single-dot task, but it did not occur in either direction on the random-dot task. Performance after practice was at very different levels on the slow single-dot and slow random-dot tasks despite comparable initial thresholds. At the faster speeds, significant practice effects occurred only in the oblique direction on the single-dot task, but they occurred in both directions on the random-dot task. There was also overlap between oblique and cardinal thresholds on the fast random-dot task, but not in the fast single-dot task. Finally, at these faster speeds, perceptual learning occurred at a significantly greater rate in the single-dot oblique condition than in the single-dot cardinal condition. Perceptual learning in oblique and cardinal directions occurred at comparable rates when the random-dot patterns were presented at $10 \% \mathrm{sec}$ or $16 \% \mathrm{sec}$. The differences between the results from Experiments 1 and 3 imply that the different practice effects reported earlier by Vogels and Orban (1985) and Ball and Sekuler (1987) cannot be attributed exclusively to the presence/absence of motion per se and that the pattern of threshold reduction for moving stimuli may also depend on the number of cues to direction.

Taken together, the results from the three experiments reported here offer support for the third hypothesis set forth in the introduction. This hypothesis states that practice effects associated with these tasks depend on both the stimulus' velocity and the number of cues to direction/ orientation. On the basis of the difference between sequential and simultaneous excitation, this hypothesis predicts that the effect of practice on single-dot direction and stationary-line orientation tasks will be similar at faster 
velocities, but different at slower velocities. The data from Experiments 1 and 2 are consistent with this prediction. The third hypothesis also predicts that different practice effects should be observed on single-dot and random-dot direction tasks of identical velocities, since the two stimulus types differ with respect to the number of directional cues. This prediction is supported by the results from Experiments 1 and 3. Unlike the first two hypotheses, which were contradicted by the practice effects observed in the present experiment, the findings across the three experiments reported here represent a converging argument for the third hypothesis.

While one can merely speculate about the physiological substrates that may have been altered during training, the difference between the practice effects observed in the velocity-matched single-dot and random-dot tasks suggests that different mechanisms may be subserving direction discrimination for these two stimuli. An alternate possibility is that performance on the two tasks may be mediated by a single motion mechanism that is sensitive to the number of cues to direction, since this was the only factor that distinguished a moving single-dot from a moving random-dot pattern. The data from Experiments 1 and 3 would be consistent with either of these possibilities, but not consistent with speculation that direction discrimination on single-dot and random-dot tasks is mediated by a single mechanism that is insensitive to the number of directional cues.

\section{GENERAL DISCUSSION}

In the present study, three experiments were conducted to assess the practice effects associated with direction and orientation discrimination tasks. The rationale for these experiments was that, by analyzing how training affects discrimination of three stimuli with very specific physical similarities and differences, the mechanism(s) underlying direction and orientation discrimination might be more clearly understood. Our results confirm and extend those found previously for perceptual learning on line-orientation discrimination tasks (Vogels \& Orban, 1985) and random-dot direction discrimination tasks (Ball \& Sekuler, 1987). The orientation-specific practice effects that Vogels and Orban (1985) reported for stationary lines subtending $15^{\circ}$ were replicated in the present study at line lengths of $5^{\circ}$ and $8^{\circ}$. However, because no significant perceptual learning was evident at either oblique or cardinal orientations when observers trained on $\mathbf{a}^{\circ}$ stimulus, the orientation-specific practice effects appear to depend on line length. This is consistent with the claim that the oblique effect decreases with decreasing line length (Vandenbussche et al., 1983; Vogels et al., 1984). The data from the faster conditions $(10 \% \mathrm{sec}$ and $16^{\circ} / \mathrm{sec}$ ) in Experiment 3 of the present study also coincide nicely with the practice effects obtained in Ball and Sekuler's (1987) random-dot direction discrimination task wherein the stimuli moved at $10 \% \mathrm{sec}$. However, these effects seem to have some speed dependency, since we found no evidence for perceptual learning in the slow random-dot task $(2 \% \mathrm{sec})$ at any direction of motion.

The experiments reported here facilitated evaluation of three different hypotheses formulated to account for the different practice effects previously shown by Vogels and Orban (1985) and Ball and Sekuler (1987). An assumption of the first hypothesis was that the differences in perceptual learning observed earlier on the orientation and direction tasks were exclusively caused by differences in the number of cues to direction and orientation. This hypothesis predicts a common pattern of threshold reduction on single-dot direction and stationary-line orientation discrimination across trajectory/line lengths, because these stimuli provide cues to direction/orientation at just one spatial location. A comparison of the results from Experiments 1 and 2 argues against this hypothesis. When the line/trajectory lengths subtended only $1^{\circ}$ of visual angle, practice significantly affected single-dot direction discrimination, but not line-orientation discrimination. A second hypothesis was based on the assumption that the different practice effects originally reported for orientation and direction discrimination were attributable solely to the presence or absence of motion. If this were the case, similar practice effects would be expected on single-dot and random-dot direction tasks, so long as both types of stimuli moved at the same velocity. An analysis of the data from Experiments 1 and 3 contradicts the second hypothesis, however, since unique patterns of threshold reduction were found on the single-dot and random-dot tasks at both fast and slow speeds. By contrast, the findings from our three experiments provide converging support for the third hypothesis, which assumes that practice effects on these tasks are determined by both the number of cues to direction/orientation and the stimulus velocity. Because the difference between simultaneous and sequential excitation of receptors on the retinal mosaic lessens with increasing speed, this hypothesis predicts similar patterns of threshold reduction on the line-orientation task and the single-dot direction task at faster speeds, but a dissimilar pattern at slower speeds. The hypothesis also predicts different practice effects for velocity-matched single-dot and random-dot direction discrimination, since the number of cues to direction differs in these two types of stimuli. The velocity-dependent similarities between the results from the single-dot and line-orientation tasks and the dissimilarities between the single-dot and random-dot data are consistent with the third hypothesis. These findings suggest that both the stimulus velocity and the number of cues to direction/orientation influence the pattern of threshold reduction.

The findings from the present study have implications for the underlying mechanisms that may have been modified during training. The similarity between practice effects in the orientation and fast single-dot experiments does not require that performance on these different tasks be mediated by a common mechanism. It is nevertheless more parsimonious to attribute the similar patterns of threshold reduction to a single mechanism than to as- 
sume that these results were produced by independent mechanisms. One might imagine an ensemble of units having relatively small receptive fields and similar orientation tuning firing simultaneously to a stationary line or nearly simultaneously to a single dot moving quickly in a particular direction. Although these characteristics might be attributed to cells in V1 or its immediate projection areas, such as V2 or MT (Van Essen \& Maunsell, 1983), we do not have the data to exclude other areas that also exhibit direction and orientation selectivity. The results from the present study do suggest that direction discrimination for velocity-matched single-dot and randomdot stimuli might not be mediated by the same mechanism, since different patterns of threshold reduction were observed on the respective tasks. Alternatively, a single motion mechanism that is sensitive to the number of cues contained in the stimulus could parsimoniously account for the different practice effects obtained in the randomdot and single-dot direction tasks. If this were the case, however, the response of this single motion mechanism would have to be adversely affected by the presence of more cues to direction-direction thresholds were consistently higher on the random-dot task than on the singledot task.

There are at least two explanations of the threshold reduction observed in the present study that do not make recourse to perceptual learning. The first is that, in the absence of a fixation point, the subjects may have learned to move their eyes more effectively during training. This implies that the practice-based improvements were motor in nature, and not perceptual. The data from our three experiments suggest that this is an unlikely possibility. If it is assumed that the significant practice effects obtained in the fast oblique conditions of the random-dot direction task were caused by improvements in the observers' abilities to conduct oblique eye movements, then significant practice effects would also be expected on the velocity-matched single-dot task. This was not the case. Practice effects also differed in the slow single-dot discrimination and short-line-orientation conditions, despite the fact that eye movements would have been of equal length and direction on the two tasks. Indeed, we feel that these data support our decision not to use a fixation point, since a visual marker may have been used to judge the direction or orientation of the stimuli.

A second account of the decline in thresholds is based on a change in "higher order" or "cognitive" processes rather than a change in perceptual processes. This, too, seems inconsistent with the pattern of threshold reduction that occurred in all three experiments reported here. If an observer who initially "misunderstood" the task suddenly learned the appropriate discrimination, then a corresponding abrupt decline in threshold would be expected. No such evidence was found. Conversely, the failure to find significant threshold reduction cannot have been due to the observers' inability to "understand" the task, since almost all trial blocks were characterized by chi-square statistics that indicated that the responses were statisti- cally indistinguishable from a cumulative normal curve. This suggests that all observers understood the task sufficiently well to consistently perform at greater than chance levels.

The failure to find significant threshold reduction in some of the experimental conditions might be attributable to a "ceiling" effect. For example, performance on the fast cardinal single-dot task and the long cardinal orientation task may have been near asymptotic levels at the start of training, whereas the potential for threshold reduction was much greater in the respective oblique conditions. Thus, differences between oblique and cardinal thresholds may simply reflect different points on the same learning curve. This seems plausible in certain cases. Inspection of Figure 1 reveals that, despite better initial performance at the cardinal directions, cardinal and oblique thresholds on the slow single-dot task overlap throughout training. Figure 7 suggests that the same is true for slow random-dot direction discrimination tasks, since oblique and cardinal thresholds overlapped during the course of training. The similarity between final oblique and final cardinal thresholds implies that a single learning curve may accurately describe the practice effects associated with each. On the other hand, Figures 2 and 5 suggest that, despite significant improvements at the oblique directions/ orientations, oblique thresholds asymptote at a level that does not overlap with even the highest cardinal thresholds. Indeed, Vogels and Orban (1985) report that JNDs in oblique orientation discrimination improved gradually over the first 2,520 trials and then stabilized at a level greater than the final cardinal JNDs regardless of the amount of additional practice. The fact that the JNDs asymptote at different points in the oblique and cardinal conditions suggests that these orientation-specific differences in discrimination do not simply represent two different points on the same learning curve. We do not attempt to distinguish whether these different asymptotic levels reflect the operation of two independent systems or the orientation-specific limitations of a single system.

Finally, all observers in the present study who demonstrated significant threshold reduction did so in a gradual manner throughout the duration of training. This time course of threshold reduction has been found on a variety of visual tasks. "Fine tuning" across sessions has been evident in line-orientation discrimination experiments (Vogels \& Orban, 1985) and random-dot direction discrimination experiments (Ball \& Sekuler, 1987). Mayer (1983) found that practice-based improvements in observers' ability to detect sinusoids at various orientations occurred over a 2-week period. Gradual improvements in both parafoveal acuity (Beard, Levi, \& Reich, 1995; Saugstadt \& Lie, 1964) and sensitivity to foveally viewed 2-point vernier stimuli (McKee \& Westheimer, 1978) have also been reported. However, other investigations have revealed evidence for a "fast learning" process characterized by significant improvements within a single session. Kumar and Glasser (1993) observed within-session improvements in nonstereo hyperacuities, and orientation- 
specific practice-based improvements have been found within a training period in several studies (Fiorentini \& Berardi, 1981; Karni \& Sagi, 1993; Poggio, Fahle, \& Edelman, 1992; Ramachandran \& Braddick, 1973). Karni and Sagi's (1993) study is unique in that within-session learning transpired only in the initial session, and performance at the beginning of each daily session was markedly better than that at the conclusion of the previous day's session. This represents a variation on the pattern of within-session threshold reduction. After analyzing threshold reduction across the ten 60 -trial blocks obtained from each observer in each session, we found only 1 observer who demonstrated significant within-session learning. Thresholds also declined significantly across sessions for this observer. Overall, our results are much more consistent with a gradual "fine tuning" of visual mechanisms rather than with "fast learning."

\section{REFERENCES}

APELle, S. (1972). Perception and discrimination as a function of stimulus orientation: The "oblique effect" in man and animals. Psychological Bulletin, 78, 266-278.

BALL, K., \& SEKULER, R. (1979). Masking of motion by broadband and filtered directional noise. Perception \& Psychophysics, 26, 206-214.

BALL, K., \& SEKULER, R. (1987). Direction-specific improvement in motion discrimination. Vision Research, 27, 953-965.

BeARD, B. L., Levi, D. M., \& Reich, L. N. (1995). Perceptual learning in parafoveal vision. Vision Research, 35, 1679-1690.
FIorentinI, A., \& Berardi, N. (1981). Learning in grating waveform discrimination: Specificity for orientation and spatial frequency. $V i$ sion Research, 21, 1149-1158.

KARNI, A., \& SAGI, D. (1993). The time course of learning a visual skill. Nature, 365, 250-252.

Kumar, T., \& GlasSER, D. A. (1993). Initial performance, learning, and observer variability for hyperacuity tasks. Vision Research, 33, 22872300.

MAYER, M. J. (1983). Practice improves adults' sensitivity to diagonals. Vision Research, 23, 547-550.

MCKeE, S. P., \& WeSTHEIMER, G. (1978). Improvement in vernier acuity with practice. Perception \& Psychophysics, 24, 258-262.

Poggio, T., Fahle, M., \& Edelman, S. (1992). Fast perceptual learning in visual hyperacuity. Science, 256, 1018-1021.

Ramachandran, V. S., \& Braddick, O. (1973). Orientation specific learning in stereopsis. Perception, 2, 371-376.

SAUGSTAD, P., \& LiE, I. (1964). Training of peripheral visual acuity. Scandinavian Journal of Psychology, 5, 218-224.

VANDEnbussche, E., Orban, G. A., \& MaEs, H. (1983). Influence of line length on the orientation discrimination of the cat. Archives Internationales de Physiologie et de Biochemie, 91, 25.

VAN ESSEN, D. C., \& MAUNSELL, J. H. (1983). Hierarchical organization and functional streams in the visual cortex. Trends in Neurosciences, 6, 370-375.

Vogels, R., \& ORBAN, G. A. (1985). The effect of practice on the oblique effect in line orientation judgments. Vision Research, 25, 1679-1687.

Vogels, R., ORban, G. A., \& Vandenbussche, E. (1984). Meridional variations in orientation discrimination in normal and amblyopic vision. Investigative Ophthalmology \& Visual Science, 25, 720-728.

(Manuscript received July 3, 1995;

revision accepted for publication February 28, 1996.) 